\title{
La enseñanza de STEM en Educación Primaria desde una perspectiva de género
}

\author{
STEM education in Primary Education from a gender perspective
}

\author{
(iD) Javier Arabit García \\ Universidad de Murcia (España) \\ (iD) María Paz Prendes Espinosa \\ Universidad de Murcia (España) \\ (iD) José Luis Serrano Sánchez \\ Universidad de Murcia (España)
}

\begin{abstract}
Resumen
Este estudio tiene como principal objetivo determinar las necesidades que 141 estudiantes y 67 docentes de siete centros de Educación Primaria tienen en relación con la enseñanza de STEM y detectar posibles diferencias y similitudes en función del género. Este objetivo forma parte del proyecto europeo CREATEskills. Desde un diseño de investigación no experimental de tipo exploratorio, se han utilizado dos cuestionarios validados a través de un doble procedimiento (juicio de expertos y estudio piloto). Los resultados indican que los docentes reclaman más medios y formación para enseñar STEM, mientras que el alumnado desea emplear recursos digitales y realizar experimentos. En relación con las diferencias de género, se deduce una visión sobre la enseñanza de STEM más crítica en las maestras que en los maestros, pero no existen diferencias significativas según el género entre el alumnado.
\end{abstract}

\begin{abstract}
The aim of this study is to identify the needs of 141 students and 67 teachers from seven Primary Education schools in relation to STEM teaching, detecting possible gender differences and similarities. This objective is part of the european CREATEskills project. On the basis of an exploratory and non-experimental research design, two validated questionnaires were used through a dual procedure (expert judgment and pilot study). The results indicate that teachers demand additional resources and training for STEM teaching, while students prefer to use digital resources and conduct experimental work. In relation to gender differences, female teachers adopted a more critical view of STEM teaching in comparison to male teachers, but no significant gender differences were identified among students.
\end{abstract}

\section{Palabras clave / Keywords}

educación, educación primaria, género, STEM, matemáticas, ciencias, tecnología education, primary education, gender, STEM, mathematics, science, technology 


\section{Introducción}

En los últimos años, han proliferado estudios sobre la enseñanza de STEM (acrónimo formado por las iniciales en inglés de las áreas de Ciencia, Tecnología, Ingeniería y Matemáticas). Estas publicaciones se centran, sobre todo, en las metodologías que se aplican o se deberían aplicar para la enseñanza y el aprendizaje de estas áreas científicas (Arabit y Prendes, 2020). Sin embargo, en paralelo, surge un creciente interés en estudiar otras implicaciones de corte más sociológico, como la perspectiva de género, es decir, la posible brecha entre hombres y mujeres en la enseñanza de STEM y las profesiones relacionadas con estas áreas (UNESCO, 2019). Un ejemplo de ello es el proyecto W-STEM sobre la brecha de género en las STEM (GarcíaHolgado et al., 2020), proyecto que se enmarca en las prioridades de la Comisión Europea para los próximos años.

En la revisión realizada por Göktepe y Şükrü (2015), los autores comprobaron cómo desde 2010 aumentaron las investigaciones sobre la enseñanza STEM. Los temas que ocupan el mayor interés en los estudios son, por este orden: el análisis de las metodologías didácticas empleadas y las diferencias entre niños y niñas tanto en el logro académico como en el nivel de ocupación en puestos profesionales asociados a STEM. En este sentido, encontramos un aumento importante en los estudios enfocados en la brecha de género en ámbitos STEM (Göktepe y Şükrü, 2015), aspecto clave en este artículo, tanto en el interés y las preferencias de los estudiantes como en la representación de la mujer en puestos de trabajos relacionados con STEM (Pasha-Zaidi y Afari, 2015; Sassler, Glass, Levitte y Michelmore, 2017).

En países como Estados Unidos surge también una especial preocupación por mejorar la enseñanza de STEM en aquellos grupos desfavorecidos, lo que se debe, según Xie, Fang y Shauman (2015), a la consideración de las STEM como una prioridad para la mejora del crecimiento económico a largo plazo. En esta misma línea, Morentin-Encina, Ballesteros y Mateus (2019) señalan la importancia de diversos factores en la enseñanza de STEM, como la influencia de la clase social, el origen étnico y el género en los resultados académicos.

Según los resultados del último informe PISA (OCDE, 2019) con estudiantes de 15 años, en España los chicos obtuvieron un resultado 6 puntos superior al de las chicas en matemáticas. Este dato coincide con la diferencia de 5 puntos, también a favor de los chicos, en la media de los países de la OCDE. Sin embargo, el rendimiento de las chicas en ciencias fue de 2 puntos por encima de los chicos. Según el estudio de la OCDE con respecto a las expectativas profesionales de los estudiantes de alto rendimiento, uno de cada tres alumnos espera trabajar como científico o ingeniero, pero solo una de cada cinco alumnas.

Dapía, Escudero y Vidal (2019) realizaron un estudio con 378 alumnos de Educación Primaria donde no encontraron diferencias relevantes entre niños y niñas en cuanto al conocimiento de las ciencias o a su deseo de llegar a ser científicos o científicas, pero constataron ciertas diferencias de género en lo referido a las actitudes, hallando mayor confianza hacia las ciencias por parte de los chicos.

Por su parte, Reinking y Martin (2018) recogen diferentes interpretaciones que pueden ser los factores causales de la falta de presencia femenina en campos STEM. En primer lugar, que los estereotipos y las prácticas de socialización en las diferentes culturas suelen girar en torno al dominio masculino. Esto se da desde la infancia, lo cual puede influir negativamente en el rendimiento académico de las niñas en asignaturas STEM desde edades tempranas. Un segundo punto de vista indica que la noción de pertenencia al grupo provoca que los estudiantes prefieran realizar las actividades similares que realizan sus grupos de iguales, aunque no coincidan ni con sus intereses, ni con sus habilidades. Esta idea concuerda con los resultados encontrados también por Raabe, Boda y Stadtfled (2019) en su investigación con casi cinco mil adolescentes en Suecia. La tercera teoría recogida por Reinking y Martin (2018) insiste de nuevo con los estereotipos, pero en esta ocasión los existentes entre los profesionales que trabajan en los campos STEM. Desde esta perspectiva, los clichés profesionales pueden ir en contra de los rasgos de la personalidad deseados para las mujeres, supuestamente más sociables y abiertas que la teóricamente incomodidad social o el carácter introvertido de los profesionales del ámbito STEM.

Blazev, Karabegovic, Burusic y Selimbegovic (2017) analizaron el nivel de creencias estereotipadas de género sobre las asignaturas relacionadas con STEM en más de 800 estudiantes de Educación Primaria de Croacia. Los hallazgos en este estudio indican una relación directa entre un alto rendimiento entre el género masculino y el apoyo a los estereotipos. Sin embargo, no se encontró relación entre los logros académicos anteriores y los estereotipos. Esta investigación concluye que es necesario centrar los esfuerzos en reducir las creencias estereotipadas entre los grupos de niños en asignaturas STEM. Teniendo en cuenta que la segregación parece producirse sobre los ocho y diez años (Reinking y Martin, 2018), resulta lógico que desde la etapa de Educación Primaria comiencen a tomarse medidas para paliar esta brecha de género en ámbitos STEM. 
Savinskaya (2017) va incluso algo más lejos y muestra en su estudio la importancia de introducir antes de la etapa de primaria programas de formación STEM que tengan en cuenta las características específicas de cómo aprenden niños y niñas para garantizar la misma motivación y actitud positiva respecto a los ámbitos STEM.

Cheryan et al. (2016) consideran que hay diversos factores que pueden explicar la brecha de género existente en las titulaciones STEM, como la carencia de experiencias en asignaturas STEM en los niveles iniciales de la enseñanza reglada, y la percepción de la autoeficacia en estas áreas. Por su parte, Kanny, Sax y RiggersPieh (2014) realizaron una revisión sistemática analizando más de trescientos artículos publicados en los últimos cuarenta años. Las barreras estructurales en Educación Primaria, los factores psicológicos y las creencias fueron las temáticas que más centraron la atención en las investigaciones revisadas que trataban de determinar los motivos por los que se produce la brecha de género en STEM. La influencia y las expectativas de las familias ha sido un factor que ha disminuido en los estudios en las dos últimas décadas. Existen interesantes estudios en la etapa de secundaria, como el realizado por Sáinz y Müller (2017), en el que participaron casi 800 estudiantes españoles. Se observó que los niños cuyas madres habían completado estudios intermedios eran más propensos a estar interesados en ámbitos STEM. De otro lado, en el caso de las niñas cuyas madres poseían estudios intermedios existe una probabilidad mayor de inclinarse por estudios relacionados con ciencias de la salud. De esta manera, se evidenció la influencia que la familia -con estudios intermedios- puede tener en las aspiraciones de los estudiantes para decantarse -o no-por ocupaciones relacionadas con los ámbitos STEM.

Baptista de Oliveira, Unbehaum y Gava (2019) seleccionaron 55 artículos que trataban sobre la igualdad de género en la enseñanza de STEM. Concluyeron que la principal preocupación de los investigadores es la mejora del desempeño de las mujeres en educación STEM. Sin embargo, parece no haber una reflexión crítica sobre igualdad de género, lo que implicaría reflexionar sobre las trayectorias sociales de hombres y mujeres, incluidos los obstáculos que no se limitan al contexto profesional (como cuidado familiar e infantil, tareas domésticas, etc.), pero obstaculizan el trabajo de las mujeres para desarrollar sus carreras en todas las áreas, particularmente en STEM.

En este sentido, Morales y Morales (2020) explican la brecha de género en STEM aludiendo a factores psicológicos (la brecha en términos de autoconcepto, autoconfianza y autoeficacia, creencias y percepciones, y diferencias en intereses y preferencias) y a factores socioculturales (por efecto de influencia parental y socialización, discriminación y sesgos, estereotipos y roles de género).

A pesar de las anteriores consideraciones y conclusiones de numerosos estudios, Sáinz $(2020$, p. 19) afirma que "no hay un plan estratégico que permita valorar desde las entidades públicas la situación y proponer medidas que pongan fin a las desigualdades entre hombres y mujeres a la hora de elegir estudios y profesiones". Según la autora, para dar respuesta a este complejo problema es necesario contar con la implicación de las administraciones, sistema educativo, empresas, medios de comunicación, familias de los estudiantes y de la sociedad en general, pues, como hemos visto, tampoco hay una única causa que nos explique las diferencias detectadas.

\section{Metodología}

\subsection{Objetivos}

Tras la revisión de literatura, se pone de manifiesto que las investigaciones demuestran la existencia de diferencias de género en relación con el ámbito de las STEM. Por este motivo, es relevante preguntarse qué ocurre con la enseñanza de estas áreas en la formación básica y obligatoria, ya que, como señalan Cheryan et al. (2016), estas etapas educativas iniciales pueden ayudarnos a explicar las diferencias que se producen con posterioridad en la vida adulta. Es por ello que se justifica la necesidad de ahondar en la realidad, para lo cual nuestra investigación se plantea los siguientes objetivos:

- Analizar las creencias de docentes y estudiantes de primaria en relación con la enseñanzaaprendizaje de STEM.

- Analizar la percepción de los docentes de primaria participantes en el estudio en relación con sus necesidades de formación y con la disponibilidad de recursos en los centros.

- Analizar las posibles diferencias de género percibidas por docentes y estudiantes en relación con la enseñanza-aprendizaje de las STEM. 
Los resultados de esta investigación serán de utilidad para el diseño de planes de formación del profesorado, tanto en los niveles iniciales como en lo relativo a la formación permanente. Del mismo modo, podrán ser útiles para replantear las metodologías y estrategias con las que se trabajan las STEM en enseñanza primaria y mejorar así, no solamente las competencias y habilidades de los estudiantes, sino su motivación, y también la reducción de las diferencias de género en este ámbito de la ciencia.

\subsection{Diseño de investigación y participantes}

Este artículo presenta los datos de una investigación exploratoria y no experimental que recoge el análisis descriptivo realizado a través de la técnica de encuesta y utilizando como instrumentos dos cuestionarios que fueron aplicados a docentes y estudiantes de Educación Primaria. Teniendo en cuenta los objetivos de la investigación y el diseño de esta, no se establecen comparaciones entre grupos.

La muestra participante se compone de 141 estudiantes y 67 docentes de 7 colegios de Educación Primaria. Los centros son elegidos por conveniencia con los criterios de ser de titularidad pública, pertenecer a la Región de Murcia (España) y tras aceptar la participación en la encuesta tanto del profesorado como del alumnado (en este último caso, con la correspondiente autorización de las familias tras ser informadas del proyecto). Aunque esta muestra es amplia e interesante para alcanzar los objetivos del estudio, no es representativa. No permite establecer inferencias, ni desarrollar conclusiones generalizables al resto de población objeto de estudio. Sin embargo, hace posible la obtención de evidencias consistentes sobre la percepción que tienen los docentes y los estudiantes de Educación Primaria en relación con las similitudes y diferencias de género en la enseñanza de STEM.

Con respecto al alumnado, el $47.5 \%$ de los participantes son chicos, mientras que el $52.5 \%$ son chicas. El rango de edad del alumnado está entre 10 y 13 años (de 5.ํ y 6. de primaria).

En cuanto al profesorado participante, el $81.5 \%$ son mujeres y el $18.5 \%$ son hombres. El $53.7 \%$ de los docentes tiene entre 35 y 50 años, un $26.9 \%$ tiene más de 50 años y un $19.4 \%$ menos de 35 (la edad media es de 42.9 años, con d.t. $=9.13$ ). En cuanto a los años de experiencia de los docentes participantes, la media es de 17.8 años (d.t.=8.48) y casi la mayoría de ellos (46.3\%) está en un intervalo de entre 11 y 20 años de experiencia.

\subsection{Instrumentos}

Teniendo en cuenta los objetivos del estudio, se hizo necesario diseñar dos cuestionarios ad hoc. Siguiendo las fases propuestas por Martin (2011), tras definir los contenidos y concretar las variables, se redactaron las preguntas y se sometieron ambos instrumentos a un procedimiento de doble validación: por una parte, con la técnica de juicio de expertos (Escobar-Pérez y Cuervo-Martínez, 2008) y, por otro lado, un piloto con una muestra de dos maestras y cinco estudiantes de 5. y 6. de primaria. En ambos cuestionarios se han introducido ítems correspondientes a las mismas dimensiones de análisis (Tabla 1 y Tabla 2) teniendo en cuenta las revisiones de estudios anteriores y los objetivos de la investigación: datos sociodemográficos; relevancia de STEM; aspectos metodológicos; recursos e infraestructuras; formación del profesorado; motivación de los estudiantes, diferencias de género y evaluación general.

El cuestionario de profesorado incluye 30 preguntas, 28 de ellas cerradas de tipo dicotómico y politómico y 2 preguntas abiertas para reflejar su opinión y sugerencias de mejora. En el caso del cuestionario para el alumnado, se redujo a 15 preguntas, siendo abierta solamente la última para recoger sus sugerencias de mejora.

\section{Tabla 1}

Relación de dimensiones, variables y correspondencia con las preguntas del cuestionario del profesorado

\begin{tabular}{cll}
\hline \multicolumn{1}{c}{ Preguntas } & \multicolumn{1}{c}{ Dimensión } & \multicolumn{1}{c}{ Variables } \\
\hline $1-2-3-4-5$ & Datos & Edad, género, país, años de experiencia como docente y asignaturas \\
& sociodemográficos & STEM o no STEM que imparte el docente \\
Relevancia de la & Importancia que el docente considera que tiene la enseñanza de STEM \\
Enseñanza de & $\begin{array}{l}\text { en primaria para la aplicabilidad de los contenidos y para el futuro de los } \\
\text { estudiantes }\end{array}$
\end{tabular}

9-10-11-12-13-14- Aspectos $15-16$ metodológicos
Tiempo dedicado a la enseñanza de STEM, metodología y recursos disponibles. 
$17-18-19-20$

21-22-23-24

25-26-27

28-29-30
Formación del

profesorado

Motivación de los

estudiantes

Diferencias de

género

Evaluación general
Formación recibida, percepción de capacitación y formación permanente

Interés, motivación y rendimiento académico

Diferencias de género en relación con la participación, motivación y el rendimiento del alumnado en materias STEM

Valoración general y aspectos de mejora sobre la enseñanza de STEM

\section{Tabla 2}

Relación de dimensiones, variables y correspondencia con las preguntas del cuestionario del alumnado

\begin{tabular}{|c|c|c|}
\hline Preguntas & Dimensión & Variables \\
\hline $1-2-3$ & $\begin{array}{l}\text { Datos } \\
\text { sociodemográficos }\end{array}$ & Edad, género, país \\
\hline $4-5$ & $\begin{array}{l}\text { Relevancia de la } \\
\text { Enseñanza de STEM }\end{array}$ & $\begin{array}{l}\text { Importancia que el estudiante considera que tienen las asignaturas } \\
\text { STEM en su vida diaria y en su futuro }\end{array}$ \\
\hline $6-7-8-9-10$ & $\begin{array}{l}\text { Aspectos } \\
\text { metodológicos }\end{array}$ & $\begin{array}{l}\text { Tiempo dedicado al aprendizaje de STEM, metodología y recursos } \\
\text { disponibles }\end{array}$ \\
\hline $\begin{array}{l}11-12-13-14 \\
15\end{array}$ & $\begin{array}{l}\text { Motivación } \\
\text { Evaluación general }\end{array}$ & $\begin{array}{l}\text { Interés, motivación, grado de satisfacción y rendimiento académico } \\
\text { Aspectos de mejora sobre la enseñanza de STEM }\end{array}$ \\
\hline
\end{tabular}

\subsection{Análisis de datos}

En el siguiente apartado mostramos los resultados más destacados tras la realización de un análisis descriptivo de cada variable (categóricas la mayoría) utilizando frecuencias. Además, se han realizado asociaciones entre variables categóricas que han permitido establecer relaciones. Para ello, se ha descrito el comportamiento conjunto de aquellas variables (de dos en dos) que se consideraron que podrían aportar información de interés, para lo cual se han utilizado tablas de contingencia. Posteriormente, se ha comprobado si existe asociación y su grado de intensidad. Para esta tarea se utiliza la prueba $\mathrm{X}^{2}$ de Pearson sobre independencia con el estadístico ji-cuadrado y la medida de asociación coeficiente de contingencia $C$, siendo el coeficiente de contingencia máximo 0.81 en todos los casos. Como criterio de selección, se han seleccionado aquellas variables que son estadísticamente significativas y muestran un grado de relación moderadamente alto (entre $60 \%$ y $75 \%$ ) tras la comparación en cada asociación del coeficiente de contingencia $C$ y el coeficiente de contingencia máximo. No se han identificado asociaciones significativas de interés con un grado de asociación alto (mayor del $75 \%$ ).

Para la realización del tratamiento y el análisis de los datos recogidos se utiliza el programa estadístico IBM SPSS (versión 24 para Windows).

\section{Resultados}

\subsection{En relación con el cuestionario del profesorado}

Los docentes contestan de forma casi unánime que es importante enseñar STEM en primaria (95.5\%) y que es clave para el futuro de los estudiantes (95.5\%). Además, consideran que es importante para que los estudiantes aprendan a aplicar los contenidos de estas materias en su vida diaria (97\%). En las tres cuestiones anteriores no hay diferencias significativas en la respuesta en función del género, ni tampoco en función de los años de experiencia o de las asignaturas que imparten.

Se pregunta a los docentes sobre el tiempo dedicado a las asignaturas STEM en el horario escolar y sus respuestas presentan diferencias de criterio. El $59.1 \%$ indica que no es suficiente y el $21.2 \%$ considera que sí lo es. Pero si filtramos los resultados según el género de los participantes, hay menos consenso: el 33.3\% de los maestros piensa que sí es suficiente el tiempo dedicado (el $50 \%$ cree que no), mientras que ese porcentaje baja al $19.2 \%$ en el caso de las maestras (el $59.6 \%$ cree que no).

El $47 \%$ de los docentes contesta que no se trabajan las materias STEM en el aula de manera diferente a como se hace en otras asignaturas (Figura 1). Si analizamos la respuesta en función del género, encontramos una diferencia importante: el $75 \%$ de los maestros asegura que trabaja las STEM de forma diferente, mientras que el porcentaje de maestras que responde de forma afirmativa a esta pregunta baja al $32.7 \%$. 


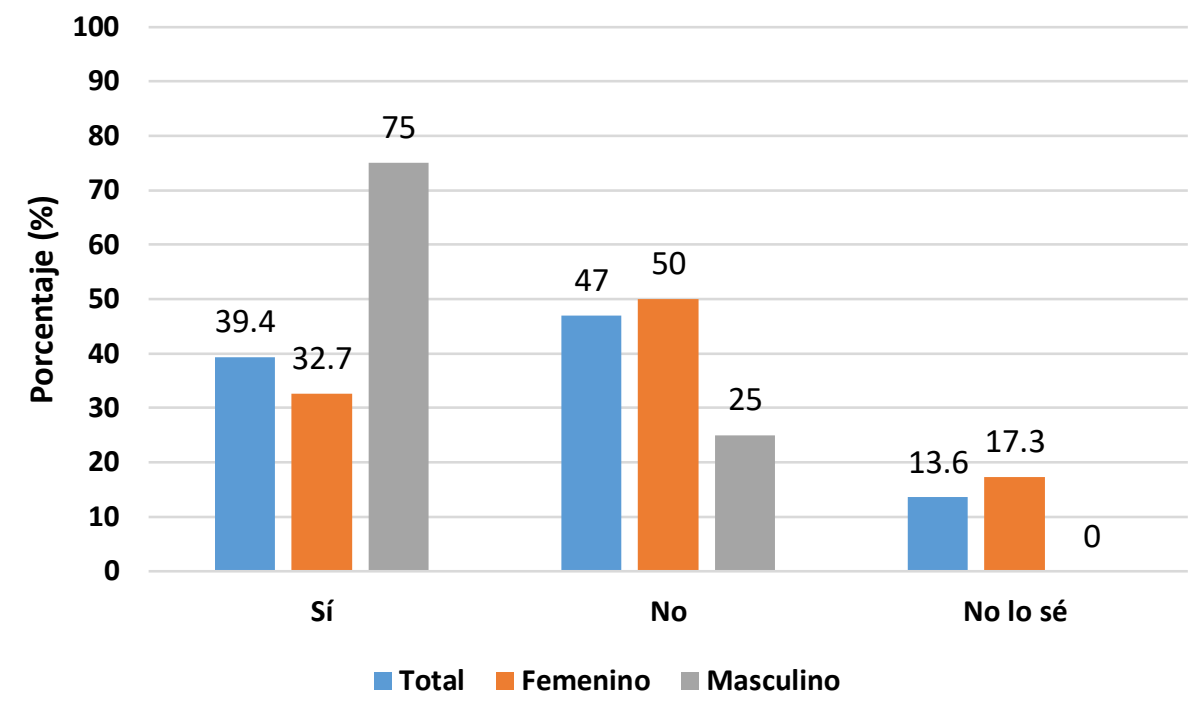

Figura 1. Respuestas del profesorado a la pregunta “¿Trabajas las asignaturas STEM de forma diferente?”

A la pregunta de si los contenidos STEM se trabajan en clase de forma integrada, un $36.4 \%$ dice que sí, otro $36.4 \%$ afirma que no y el $27.3 \%$ restante responde que no lo sabe. En esta pregunta también hay diferencias dependiendo del género: la respuesta afirmativa es del $30.8 \%$ en el caso de las mujeres y sube al $58.3 \%$ en los hombres.

Un $41.5 \%$ considera que la forma en que se enseñan las materias STEM no motiva a los estudiantes y un $30.8 \%$ piensa que sí. También en este caso hay diferencia notable según el género: un $50 \%$ de los maestros piensa que sí y solo el $25 \%$ de las maestras responde afirmativamente. La mayoría de los docentes (62.1\%) afirma que no le gusta la forma en la que se enseñan las materias STEM. En la respuesta negativa no hay una diferencia significativa entre hombres y mujeres, sin embargo, en la respuesta afirmativa sí la hay: al $33.3 \%$ de los maestros le gusta la forma de enseñar las STEM, mientras que solo el $13.5 \%$ de las maestras responde de forma positiva a esta cuestión.

Maestros y maestras opinan de forma mayoritaria que en la escuela no hay suficientes recursos materiales y tecnológicos $(81.8 \%)$ ni espacios específicos $(94 \%)$, como laboratorios o aulas de ordenadores, para trabajar de forma adecuada las asignaturas STEM con el alumnado (Figura 2).

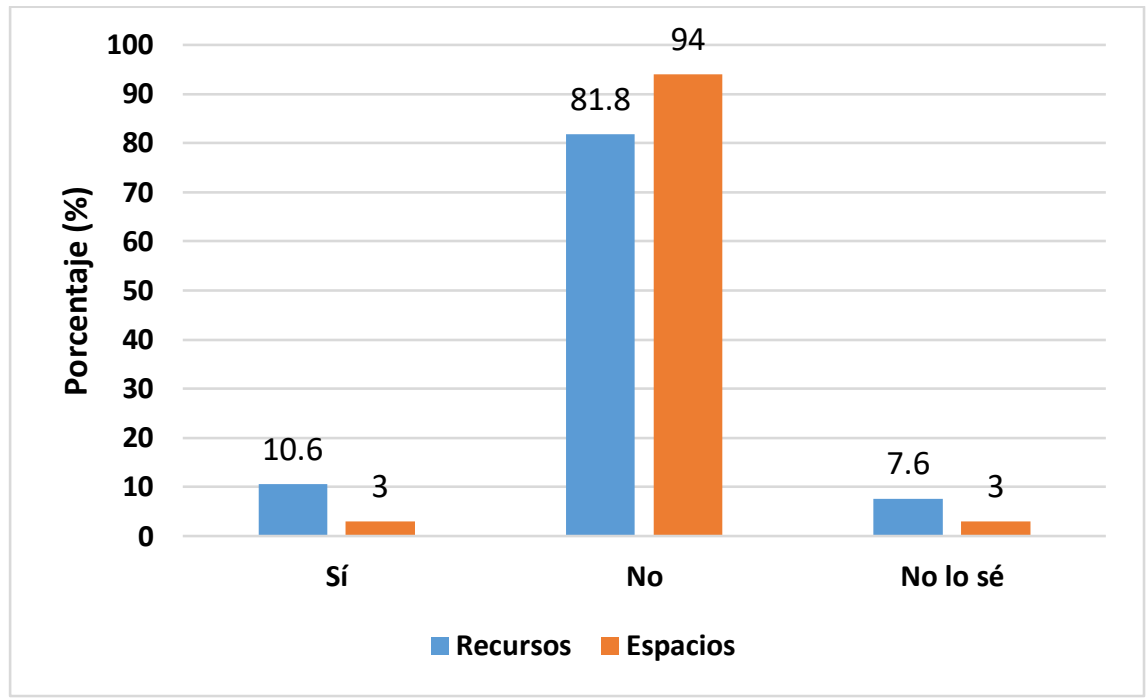

Figura 2. Respuestas del profesorado a la pregunta “Cuentas con suficientes recursos y espacios para trabajar las STEM?” 
Los siguientes resultados se refieren a diferentes aspectos relacionados con la formación. Un $62.1 \%$ del profesorado considera que actualmente no está suficientemente capacitado para trabajar las materias STEM de forma adecuada en el aula. Si analizamos la respuesta dependiendo del género, la diferencia es relevante: el $50 \%$ de los maestros creen que sí están suficientemente formados, pero solo el $15.4 \%$ de las maestras responde que sí.

El $43.9 \%$ de los participantes dice no saber si sus compañeros están suficientemente formados para enseñar STEM, el $28.8 \%$ dice que sí lo están y el $27.3 \%$ responde que no. Hay diferencia en función del género en la respuesta negativa a esta pregunta: el $30.8 \%$ de las mujeres y el $16.7 \%$ de los hombres responden que la formación de sus compañeros no es suficiente.

Casi todos los participantes (93.9\%) indican que les gustaría recibir más formación para poder enseñar mejor las materias relacionadas con STEM. A aquellos que han respondido de forma afirmativa a esta cuestión se les pide que concreten en qué aspectos les gustaría mejorar su formación. La mayoría responde que le gustaría mejorar su formación en relación con los recursos (86.4\%) y las metodologías $(81.8 \%$ ) que se pueden emplear y aplicar con asignaturas STEM. Más equilibrada es la respuesta cuando preguntamos si desean ampliar su formación para lograr una mejor motivación del alumnado: el 54.5\% responde que sí (Figura 3). En este aspecto, encontramos una gran diferencia dependiendo de si responde una maestra o un maestro: mientras que el $51.9 \%$ de ellas indica que sí le gustaría mejorar su formación para motivar al alumnado, solo el $25 \%$ de ellos responde de forma afirmativa.

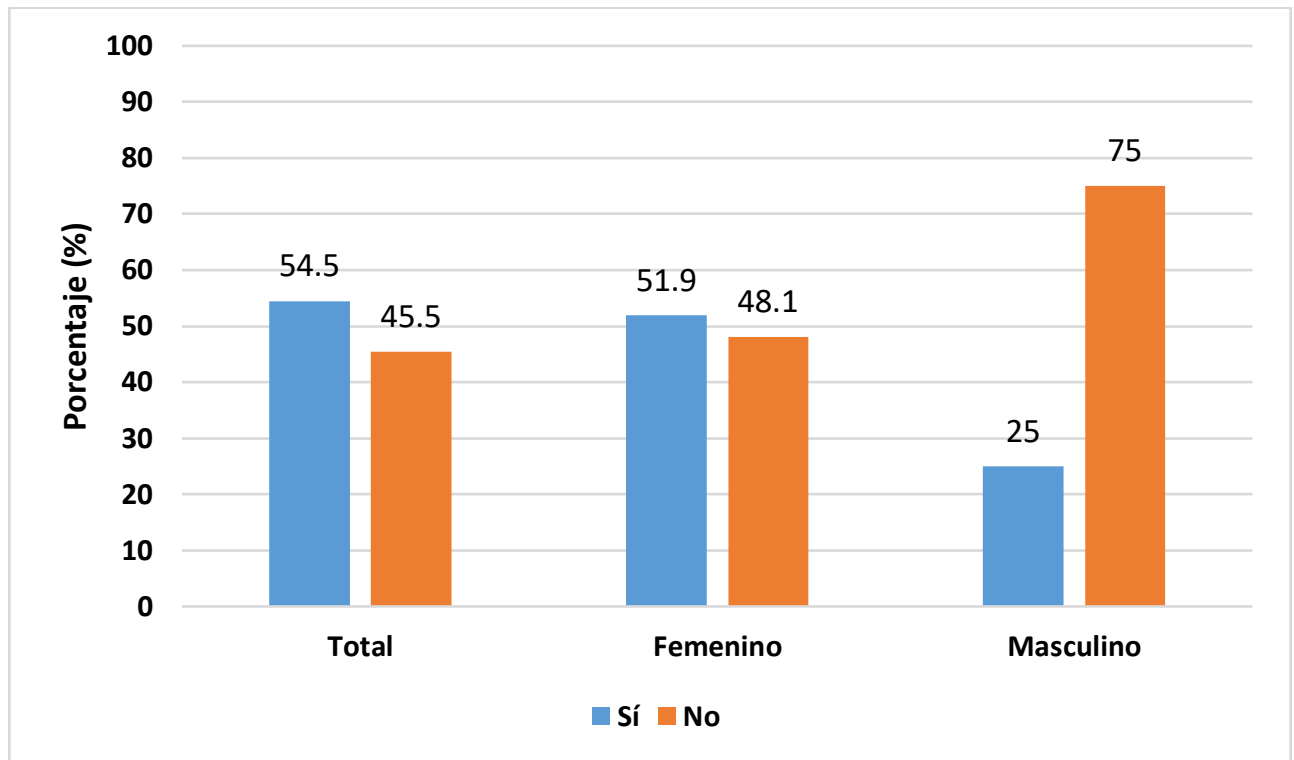

Figura 3. Respuestas del profesorado a la pregunta “ ¿Te gustaría mejorar su formación en STEM para incentivar la motivación del alumnado?”

En relación con la motivación del alumnado, una gran parte de los participantes (78.1\%) cree que sus estudiantes están motivados cuando se trabajan los contenidos STEM en el aula y califican esa motivación con 6.5 de media en una escala del 0 al 10 (d.t.=1,90). Si calculamos la calificación en función del género, tenemos un 6.3 por parte de las maestras y un 7.3 por parte de los maestros.

El $60.6 \%$ del profesorado indica que los estudiantes se esfuerzan cuando trabajan los contenidos STEM, pero encontramos una diferencia importante dependiendo del género de los participantes: opinan de ese modo el $91.7 \%$ de ellos y esa cifra se reduce al $53.8 \%$ en el caso de las maestras. La calificación media que otorgan los docentes a ese esfuerzo del alumnado es de 6.6 con d.t. $=2.56$.

Con respecto al rendimiento académico de los estudiantes en las asignaturas STEM (Tabla 3), el $53.1 \%$ de los docentes considera que es bueno y el $28.1 \%$ piensa que no. También encontramos una diferencia muy relevante en este aspecto en función del género: el $91.7 \%$ de los maestros asegura que el rendimiento del alumnado en las materias STEM es bueno, mientras que solo el $44.2 \%$ de las maestras lo cree así.

\section{Tabla 3}


Porcentajes de actuación académica del alumnado, según el profesorado

\begin{tabular}{lcrr}
\hline Género & \multicolumn{2}{c}{ ¿Es buena la actuación académica de los alumnos? } \\
& Sí & \multicolumn{3}{c}{ No lo sé } \\
\hline Femenino & 44.2 & 32.7 & 23.1 \\
Masculino & 91.7 & 8.3 & 0.0 \\
Total & 53.1 & 28.1 & 18.8 \\
\hline
\end{tabular}

La mayoría del profesorado (58.5\%) piensa que sus estudiantes están interesados en continuar su formación en competencias relacionadas con las materias STEM, aunque hay un porcentaje importante (38.5\%) que dice no saberlo. En este caso no hay diferencias significativas según el género. En cuanto a la concepción que tiene el profesorado sobre las diferencias que pudieran existir entre alumnos y alumnas en las asignaturas STEM, la mayoría de los docentes considera que no existe diferencia según el género del alumnado en los niveles de participación (76.9\%), de motivación (75.4\%) y de rendimiento académico $(76.9 \%)$. En estos aspectos no encontramos grandes diferencias dependiendo de la edad de los participantes o de si responden maestros o maestras.

Por último, se pide a los docentes que califiquen, en una escala de 1 a 5 , la calidad de la enseñanza de STEM en España. En este rango, los participantes dan una calificación media de 2.8 (d.t. $=0,77$ ) a la enseñanza de las materias científicas en Educación Primaria en nuestro país. Si filtramos la calificación media según el género de los docentes, las maestras otorgan un aprobado justo a las STEM en España con un 3.0, mientras que los maestros bajan esa calificación media a un 2.6.

Además del análisis descriptivo, hemos realizado un estudio correlacional. Respecto a las asociaciones entre variables categóricas del cuestionario del profesorado que están relacionadas, encontramos en primer lugar la relación entre los docentes que consideran que es importante enseñar STEM en primaria y que es clave para el futuro de los estudiantes (Tabla 4).

\section{Tabla 4}

Ji-cuadrado y coeficiente de contingencia $C$ entre la importancia de la enseñanza STEM en primaria y de los contenidos en el futuro.

¿Consideras importante enseñar STEM desde la escuela primaria?

$\mathbf{X}^{2} \quad \mathrm{C}$

¿Crees que los aprendizajes de los

contenidos de STEM son importantes para el

futuro de sus estudiantes?

$$
X^{2}(2,66)=31.794^{a * *}
$$

Nota: ${ }^{* *} p<.001 ; C=$ Coeficiente de Contingencia

En segundo lugar, observamos que los docentes no están satisfechos con la forma en la que se enseña las materias STEM, lo cual influye negativamente con la motivación de los estudiantes. En este caso, también existe un grado moderadamente alto de asociación (Tabla 5).

\section{Tabla 5}

Ji-cuadrado y coeficiente de contingencia $C$ entre la forma de enseñar materias STEM y la motivación de los estudiantes

¿Crees que la forma en que se enseñan las materias STEM motiva a los estudiantes?

$\mathrm{X}^{2}$

C

¿Te gusta la forma en que se enseñan las materias STEM en la escuela primaria?

$X^{2}(2,65)=30.725^{a * \star *}$

Nota: ${ }^{* * *} p<.001 ; C=$ Coeficiente de Contingencia 
La última relación detectada en este cuestionario con un grado de asociación moderadamente alto nos indica que la disponibilidad de suficientes recursos en el centro para trabajar las STEM influye en la importancia que los docentes dan a la aplicación de los contenidos STEM a la vida diaria de los estudiantes (Tabla 6).

\section{Tabla 6}

Ji-cuadrado y coeficiente de contingencia $C$ entre los recursos disponibles y la aplicación práctica del contenido STEM

¿Tiene tu escuela suficientes recursos para trabajar en las materias STEM adecuadamente?

$\mathrm{X}^{2}-\mathrm{C}$

¿Crees que es necesario que los estudiantes
aprendan a aplicar el contenido STEM a su
vida diaria?

Nota: ${ }^{\star \star \star} p<.001 ; C=$ Coeficiente de Contingencia

\subsection{En relación con el cuestionario del alumnado}

De forma muy mayoritaria, y sin diferencias significativas según el género, el alumnado cree que las ciencias y las matemáticas son importantes en su vida diaria $(87.1 \%)$ y para su futuro $(93.6 \%)$. Por otro lado, el $51.8 \%$ afirma que en las clases de estas materias no se trabaja de forma distinta a como lo hacen en otras asignaturas (sólo un $30.2 \%$ de los estudiantes dice que sí).

A la pregunta de si comprenden bien las explicaciones del profesorado, el $81.3 \%$ del alumnado responde que sí. En este caso tampoco hay una diferencia significativa entre lo que expresan los niños (80.3\%) y las niñas $(82.2 \%)$.

Con respecto a la frecuencia con que trabajan de determinadas formas en las asignaturas STEM, tampoco se obtienen resultados con diferencias destacables en función del género del alumnado. La mayoría de los alumnos responde que casi siempre realizan trabajo individual y que solo a veces realizan trabajo en grupo. Además, un $67.4 \%$ del alumnado responde que casi nunca se realizan experimentos en clase de materias STEM. En la Tabla 7 recogemos los resultados del tipo de actividades que realizan y la frecuencia.

\section{Tabla 7}

Porcentaje de empleo de distintas formas de trabajo en STEM, según el alumnado

\begin{tabular}{lrrr}
\multicolumn{1}{c}{ Forma de trabajo } & Casi nunca & A veces & Casi siempre \\
\hline Trabajo individual & 1.4 & 23.6 & 75.0 \\
Trabajo en grupo & 31.2 & 58.0 & 10.8 \\
Ejercicios prácticos en clase & 23.7 & 31.1 & 45.2 \\
El profesor explica en clase y manda deberes para & 16.5 & 27.4 & 56.1 \\
hacer en casa & & & \\
Experimentos & 67.4 & 32.6 & 0 \\
\hline
\end{tabular}

De forma análoga, como mostramos en la Tabla 8 , se pregunta al alumnado qué recursos y espacios se usan en las clases de ciencias y matemáticas. De este ítem, en el que tampoco se encuentran diferencias reseñables desde una perspectiva de género, podemos destacar que el $52.5 \%$ del alumnado afirma que casi siempre se emplea la pizarra digital, el $97.2 \%$ responde que casi nunca usan tabletas, el $97.1 \%$ dice que casi nunca trabajan en el laboratorio y un porcentaje destacable dice que a veces trabajan los contenidos STEM en visitas culturales y excursiones.

\section{Tabla 8}

Porcentaje de recursos y espacios empleados en asignaturas STEM, según el alumnado 


\begin{tabular}{lrrr}
\hline \multicolumn{1}{c}{ Recursos y espacios } & Casi nunca & A veces & Casi siempre \\
\hline Pizarra digital & 16.6 & 30.9 & 52.5 \\
Tabletas & 97.2 & 1.4 & 1.4 \\
Ordenadores portátiles & 74.3 & 19.3 & 6.4 \\
Sala de ordenadores & 50.4 & 45.3 & 4.3 \\
Laboratorio & 97.1 & 2.1 & 0.8 \\
Biblioteca & 67.9 & 28.5 & 3.6 \\
Visitas culturales & 37.4 & 61.2 & 1.4 \\
Excursiones & 17.8 & 72.9 & 9.3 \\
\hline
\end{tabular}

Los siguientes resultados se refieren a la motivación, esfuerzo y participación de los estudiantes en las clases de STEM. El 48.2\% del alumnado dice estar motivado en las clases de Ciencias y Matemáticas, mientras que un $34.4 \%$ no lo está (el $14.4 \%$ responde que no lo sabe). En este ítem sí encontramos leves diferencias en función del género: el $52.2 \%$ de los niños indica que están motivados, mientras que la cifra baja al $44.4 \%$ en el caso de las niñas. Cuando se les pide que califiquen en una escala de 0 a 10 su nivel de motivación, la media es de 6.25 con d.t. $=2.54$.

Con respecto a la satisfacción que tiene el alumnado con su nivel de esfuerzo (tabla 9), el $68.6 \%$ de los participantes responde que sí está satisfecho, existiendo también una pequeña diferencia entre niños $(66.1 \%)$ y niñas (74.3\%). La calificación media que ofrecen los estudiantes a su nivel de esfuerzo es de 7.83 con d.t. $=1.80$. Los participantes responden de forma mayoritaria que sí están satisfechos en su nivel de participación (72.3\%) y con sus resultados académicos (71.4\%) en las clases de STEM, con un resultado muy similar entre niños y niñas. Califican su nivel de participación con un 7.19 (d.t.=2,47).

\section{Tabla 9}

Porcentaje de satisfacción del alumnado con respecto a su motivación, esfuerzo y participación en STEM

\begin{tabular}{lccccccccc} 
& \multicolumn{3}{c}{ Motivación } & \multicolumn{3}{c}{ Esfuerzo } & \multicolumn{3}{c}{ Participación } \\
& Sí & No & No lo sé & Sí & No & No lo sé & Sí & No & No lo sé \\
\hline General & 48.2 & 37.4 & 14.4 & 68.6 & 15.7 & 15.7 & 72.3 & 14.9 & 12.8 \\
Femenino & 44.4 & 36.1 & 19.4 & 74.3 & 13.5 & 12.1 & 72.9 & 14.8 & 12.1 \\
Masculino & 52.2 & 38.8 & 8.9 & 66.1 & 18.1 & 19.6 & 71.6 & 14.9 & 13.4 \\
\hline
\end{tabular}

Entre las variables de este cuestionario solamente existe una relación con un grado moderadamente alto de asociación. En concreto, el recurso del laboratorio empleado por los estudiantes en las clases STEM se relaciona con la importancia que el alumnado otorga a los contenidos científicos para el futuro y con la comprensión correcta de las explicaciones del profesorado cuando enseñan estas materias (Tabla 10).

\section{Tabla 10}

Ji-cuadrado y coeficiente de contingencia $C$ entre el uso del laboratorio, la comprensión de las explicaciones del profesorado y la importancia de las asignaturas STEM en el futuro

Uso del laboratorio como recursos utilizado en materias STEM

\begin{tabular}{llc} 
& $\mathbf{X}^{2}$ & $\mathbf{C}$ \\
\hline $\begin{array}{l}\text { ¿Crees que las asignaturas STEM son } \\
\text { importantes para tu futuro? }\end{array}$ & $X^{2}(4,140)=46.143^{\text {*** }}$ & .498 \\
$\begin{array}{l}\text { ¿Comprendes bien las explicaciones del } \\
\text { profesorado cuando enseña materias STEM? }\end{array}$ & $X^{2}(4,138)=25.888^{\text {a }}$ (** & .397 \\
\hline
\end{tabular}

Nota: ${ }^{* *} p<.001 ; C=$ Coeficiente de Contingencia

\section{Discusión y conclusiones}


La principal finalidad de este estudio es detectar las posibles necesidades que tanto los docentes como los estudiantes de Educación Primaria participantes en la investigación tienen en relación con la enseñanza de STEM. Numerosos estudios (Blazev et al., 2017; Raabe et al., 2019; Reinking y Martin, 2018; Valle et al., 2016) muestran cómo existe una preocupación importante por detectar y paliar la discriminación que la mujer tiene en ámbitos profesionales STEM, cuyo origen parece residir por la combinación de diferentes teorías y que, según Reinking y Martin (2018), comienza a producirse entre los 8 y 10 años, rango de edad que forma parte de esta investigación. Por este motivo, en nuestra investigación hemos considerado importante la inclusión del análisis de las posibles diferencias género y así poder tenerlas en cuenta en las futuras líneas de actuación, puesto que la falta de experiencia temprana en asignaturas STEM es uno de los factores que explica la brecha de género en ámbitos STEM (Cheryan, Ziegler, Montoya y Jiang, 2016; Thibaut, Knipprath, Dehaene y Depaepe, 2018). La Educación Primaria ha sido la etapa en la que se ha centrado este estudio, aunque autores como Savinskaya (2017) consideran que, para garantizar una motivación y actitud positiva hacia los ámbitos STEM y basada en la igualdad de oportunidades entre hombres y mujeres, la formación en STEM debe iniciarse antes de Primaria.

Los docentes participantes en el estudio opinan que la enseñanza de STEM es importante para la vida diaria y el futuro de sus estudiantes, ideas que éstos comparten junto a estudios como los de Mustafa, Ismail, Tasir y Said (2016), English (2017), Murphy, MacDonald, Danaia y Wang (2018) y Sanmartí y Márquez (2017).

Maestras y maestros coinciden de forma mayoritaria en la necesidad de mejorar la formación del profesorado y de contar con más recursos (humanos, espaciales, materiales y tecnológicos) para poder impartir estas asignaturas de forma adecuada en las aulas. Este aspecto es de especial relevancia, puesto que, según Dawne (2016), la percepción que tengan los docentes sobre la enseñanza de STEM está intrínsecamente vinculada con la eficacia de su enseñanza. Esta necesidad de formación coincide por la detectada en numerosos estudios (Göktepe y Şükrü, 2015; Sanmartí y Márquez, 2017; Shernoff, Sinha, Bressler y Ginsburg, 2017; Thibaut at al., 2018).

Los docentes, en su mayoría, afirman no estar satisfechos con su preparación en STEM, la manera que tienen de enseñar estas materias y la forma en la que motivan a sus estudiantes. Sin embargo, el alumnado sí dice comprender bien las explicaciones y estar motivado, algo más los niños que las niñas, coincidiendo con los resultados obtenidos por Valle et al. (2016). En este sentido, se encuentran diferencias importantes en la percepción que tienen maestras y maestros. Ellas parecen ser más críticas, puesto que afirman que se sienten menos capacitadas y necesitarían mejorar más su formación tanto en el uso de recursos como en el conocimiento de distintas metodologías y estrategias para mejorar la motivación de los estudiantes. En general, los maestros manifiestan una visión más optimista o positiva -lo que no significa que sea más o menos realista- que las maestras en diversos aspectos de gran calado que hemos detallado: cuando se les pregunta si realizan un trabajo diferenciado e integrado de las STEM, cuando valoran los niveles de motivación, esfuerzo y rendimiento académico del alumnado, o en consideraciones relativas a la formación docente.

No obstante, esta circunstancia contrasta con el resultado que obtenemos en el ítem donde se pide a los docentes que califiquen la calidad de la enseñanza de las STEM en España: las maestras la aprueban, mientras que los maestros la califican con suspenso con cuatro décimas menos que ellas. Si bien la diferencia estadística no es muy significativa, llama la atención este cambio de tendencia: ellos son más positivos como norma general, pero la valoración que hacen sobre la enseñanza de STEM es más negativa que la de ellas. Retomando los aspectos metodológicos de la enseñanza de STEM, los estudiantes hacen un uso escaso de recursos como la tableta, el laboratorio o los ordenadores portátiles. Por el contrario, hacen más uso de la pizarra digital (aunque no podemos afirmar si la usan ellos o los docentes) y las visitas culturales y excusiones. Los docentes, por su parte, afirman que no disponen de recursos ni de espacios suficientes para una adecuada enseñanza de STEM. En este sentido, es destacable la consideración que realiza Thibaut et al. (2018) cuando indica que las escuelas con recursos limitados no tienen por qué tener menor éxito en la enseñanza STEM que otros centros bien dotados. Si tenemos en cuenta los resultados obtenidos en relación con la forma en la que trabajan los estudiantes (de manera individual, reciben explicaciones en clase y hacen deberes en casa) se observa que los docentes tienen un espacio amplio de mejora para integrar otro tipo de metodologías activas como las propuestas por Sanmartí y Márquez (2017) y Thibaut et al. (2018) y la integración de las tecnologías más allá del uso de la pizarra digital.

De todo lo anterior, se concluye que, dado que se han hallado diferencias (algunas de ellas significativas) en función del género de los participantes, además de tener en cuenta los aspectos fundamentales en los que hay consenso (como la falta de recursos y de espacios específicos o el uso escaso de las tecnologías), se 
deberá tener en consideración la perspectiva de género -sobre todo entre el profesorado- en el momento de diseñar e implementar experiencias de enseñanza de STEM.

Además, sería interesante valorar el papel que las familias pueden tener, así como el contexto socioeconómico y cultural, tal y como concluyen Baptista de Oliveira, Unbehaum y Gava (2019), Morales y Morales (2020), Sáinz (2020), Sáinz y Müller (2017) o Xie et al. (2015), quienes aluden a la influencia determinante de estos factores en la predicción del éxito en el aprendizaje de STEM. Es preciso poner en marcha propuestas para la intervención (Sáinz, 2020) que den respuesta a la brecha de género en la enseñanza y el aprendizaje de STEM, con la imprescindible colaboración de toda la comunidad educativa (administración, estudiantes, docentes, familias y otros profesionales) para asegurar la igualdad de oportunidades de los estudiantes (Holmlund, Lesseig y Slavit, 2018).

Por último, en cuanto a la transferencia de conocimiento resultante de la investigación realizada, y enlazando con la necesidad -manifestada por el profesorado- de contar con más recursos específicos para trabajar las STEM, cabe destacar que en el marco del proyecto europeo CREATEskills ${ }^{1}$ se ha creado una página web que incluye una plataforma para promover el intercambio de experiencias y actividades (para profesorado y familias), un libro guía para profesorado y una colección de materiales y actividades para trabajar numerosos contenidos mediante metodologías activas. Toda esta información está en acceso abierto y la plataforma es de uso libre mediante registro.

\section{Apoyos}

Este estudio está vinculado al proyecto "CREATEskills - Social Learning for STEM (Science, Technology, Engineering \& Mathematics) in Primary Education", financiado en el marco del programa europeo Erasmus+ (2017-1-PT01-KA201035981).

\section{Referencias}

Arabit, J. y Prendes, M. P. (2020). Metodologías y Tecnologías para enseñar STEM en Educación Primaria: análisis de necesidades. Pixel-Bit: Revista de Medios y Educación, 57, 107-128. Doi: https://doi.org/10.12795/pixelbit.2020.i57.04 Baptista de Oliveira, E. R., Unbehaum, S., y Gava, T. (2019). STEM education and gender: a contribution to discussions in Brazil. Cadernos de Pesquisa, 49(171), 130-159. Doi: https://doi.org/10.1590/198053145644

Blazev, M., Karabegovic, M., Burusic, J. y Selimbegovic, L. (2017). Predicting gender-STEM stereotyped beliefs among boys and girls from prior school achievement and interest in STEM school subjects. Social Psychology of Education, 20(4), 831-847. Doi: https://doi.org/10.1007/s11218-017-9397-7

Dawne, B. (2016). The reality of STEM education, design and technology teachers' perceptions: a phenomenographic study. International Journal of Technology and Design Education, 26(1), 61-79. Doi: https://doi.org/10.1007/s10798-0159300-9

Cheryan, S., Ziegler, S. A., Montoya, A. K., y Jiang, L. (2016). Why Are Some STEM Fields More Gender Balanced Than Others? Psychological Bulletin, 143(1), 1-35. Doi: http://dx.doi.org/10.1037/bul0000052

Dapía, M., Escudero-Cid, R. y Vidal, M. (2019) ¿Tiene género la ciencia? Conocimientos y actitudes hacia la Ciencia en niñas y niños de Educación Primaria. Revista Eureka sobre Enseñanza y Divulgación de las Ciencias 16(3), 3201. Doi: 10.25267/Rev Eureka ensen divulg cienc.2019.v16.i3.3302

English, L. D. (2017). Ādvancing elementary and middle school STEM education. International Journal of Science and Mathematics Education, 15(1), 5-24. Doi: https://doi.org/10.1007/s10763-017-9802-x

Escobar-Pérez, J. y Cuervo-Martínez, A. (2008). Validez de contenido y juicio de expertos: una aproximación a su utilización. Avances en medición, 6, 27-36. Recuperado de: https://bit.ly/2BXXFms

García-Holgado, A., Verdugo-Castro, S., González, C., Sánchez-Gómez, M.C. y García-Peñalvo, F.J. (2020). European Proposals to Work in the Gender Gap in STEM: A Systematic Analysis. IEEE Revista Iberoamericana de Tecnologías del Aprendizaje, 15(3), 215-224. Doi: 10.1109/RITA.2020.3008138

Göktepe, S. y Şükrü, A. (2015). A content Analysis Study About Stem Education. TOJET: The Turkish Online Journal of Educational Technology, 14-21. Recuperado de: https://bit.ly/2ZolP0R

Holmlund, T., Lesseig, K. y Slavit, D. (2018). Making sense of STEM education in K-12 contexts. International Journal of STEM Education, 5(32), 2-18. Doi: https://doi.org/10.1186/s40594-018-0127-2

Kanny, M.A., Sax, L.J. y Riggers-Pieh, T.A. (2014). Investigating Forty Years of STEM Research: How Explanations for the Gender Gap Have Evolved Over Time. Journal of Women and Minorities in Science and Engineering, 20(2), 127148. Doi: https://doi.org/10.1615/JWomenMinorScienEng.2014007246

Martín, B. (2011). Técnicas e instrumentos de recogida de investigación. En S. Cubo, B. Martín y J.L. Ramos (Eds.), Métodos de investigación y análisis de datos en ciencias sociales y de la salud (pp. 173-233). Madrid: Pirámide.

\footnotetext{
${ }^{1}$ Web del proyecto CREATESkills: http://createskills.eu/
} 
Morales Inga, S. y Morales Tristán, O. (2020). ¿Por qué hay pocas mujeres científicas? Una revisión de literatura sobre la brecha de género en carreras STEM. Revista Internacional de Investigación en Comunicación aDResearch ESIC, 22, 118-133. Doi: https://doi.org/10.7263/adresic-022-06

Morentin-Encina, J., Ballesteros, B., y Mateus, S. (2019). ¿Igualdad de oportunidades? Más que el mero acceso: narrativas de jóvenes con trayectorias de fracaso y abandono temprano de la educación en España y Portugal. Revista Fuentes, 21 (2), 143-159. Doi: http://dx.doi.org/10.12795/revistafuentes.2019.v21.i2.01

Murphy, S., MacDonald, A., Danaia, L. y Wang, C. (2018). An analysis of Australian STEM education strategies. Policy Futures in Education O(0), 1-18. Doi: http://dx.doi.org/10.1177/1478210318774190

Mustafa, N., Ismail, Z., Tasir, Z. y Said, M.N. (2016). A Meta-Analysis on Effective Strategies for Integrated STEM Education. Advanced Science Letters, 22(12), 4225-4229. Doi: https://doi.org/10.1166/asl.2016.8111 OCDE (2019), PISA 2018 Results (Volume I): What Students Know and Can Do. París: PISA, OECD Publishing. Doi: https://doi.org/10.1787/5f07c754-en

Pasha-Zaidi, N. y Afari, E. (2015). Gender in STEM Education: an Exploratory Study of Student Perceptions of Math and Science Instructors in the United Arab Emirates. International Journal of Science and Mathematics Education, 13(3), 1215-1231. Doi: https://doi.org/10.1007/s10763-015-9656-z

Raabe, I.J., Boda, Z. y Stadtfled, C. (2019). The Social Pipeline: How Friend Influence and Peer Exposure Widen the STEM Gender Gap. Sociology of Education, 20(10), 1-19. Doi: https://doi.org/10.1177/0038040718824095

Reinking, A. y Martin, B. (2018). The Gender Gap in STEM Fields: Theories, Movements, and Ideas to Engage Girls in STEM. Journal of New Approaches in Educational Research, 7(2), 160-166. Doi: https://doi.org/10.7821/naer.2018.7.271 Sáinz, M. (2020). Brechas y sesgos de género en la elección de estudios STEM: ¿Por qué ocurren y cómo actuar para eliminarlas? Colección Actualidad (Centro de Estudios Andaluces), 84, 1-22. Recuperado de: https://bit.ly/390fkWE Sáinz, M. y Müller, J. (2018) Gender and family influences on Spanish students' aspirations and values in stem fields. International Journal of Science Education 40(2), 188-203. Doi: https://doi.org/10.1080/09500693.2017.1405464 Sanmartí, N. y Márquez, C. (2017). Aprendizaje de las ciencias basado en proyectos: del contexto a la acción. Ápice: revista de educación científica, 1(1), 3-16. Doi: https://doi.org/10.17979/arec.2017.1.1.2020

Sassler, S., Glass, J., Levitte, Y. y Michelmore, K.M. (2016). The missing women in STEM? Assessing gender differentials in the factors associated with transition to first jobs, Social Science Research, 63, 192-208. Doi: http://dx.doi.org/10.1016/j.ssresearch.2016.09.014

Savinskaya, O.B. (2017). Gender Equality in Preschool STEM Programs as a Factor Determining Russia's Successful Technological Development. Russian Education \& Society, 59(4), 206-216. Doi:

https://doi.org/10.1080/10609393.2017.1399758

Shernoff, D., Sinha, S., Bressler, D.M. y Ginsburg, L. (2017). Assessing teacher education and professional development needs for the implementation of integrated approaches to STEM education. International Journal of STEM Education,4(13), 1-16. Doi: https://doi.org/10.1186/s40594-017-0068-1

Thibaut, L. Knipprath, H., Dehaene, W. y Depaepe, F. (2018). The influence of teachers' attitudes and school context on instructional practices in integrated STEM education. Teaching and Teacher Education, 71, 190-205. Doi: https://doi.org/10.1016/j.tate.2017.12.014

UNESCO (2019). Descifrar el código: la educación de las niñas y las mujeres en ciencias, tecnología, ingeniería y matemáticas (STEM). París: Organización de las Naciones Unidas para la Educación, la Ciencia y la Cultura. Recuperado de: https://bit.ly/3gY6qeQ

Valle, A., Regueiro, B., Piñeiro, I., Sánchez, B., Freire, C. y Ferradás, M. (2016). Actitudes hacia las matemáticas en estudiantes de Educación Primaria: Diferencias en función del curso y del género. European Journal of Investigation in Health, Psychology and Education, 6(2), 119-132. Doi: https://doi.org/10.30552/ejihpe.v6i2.161

Xie, Y., Fang, M. y Shauman, K. (2015). STEM Education. Annual Review of Sociology, 41, 331-357. Doi: https://doi.org/10.1146/annurev-soc-071312-145659

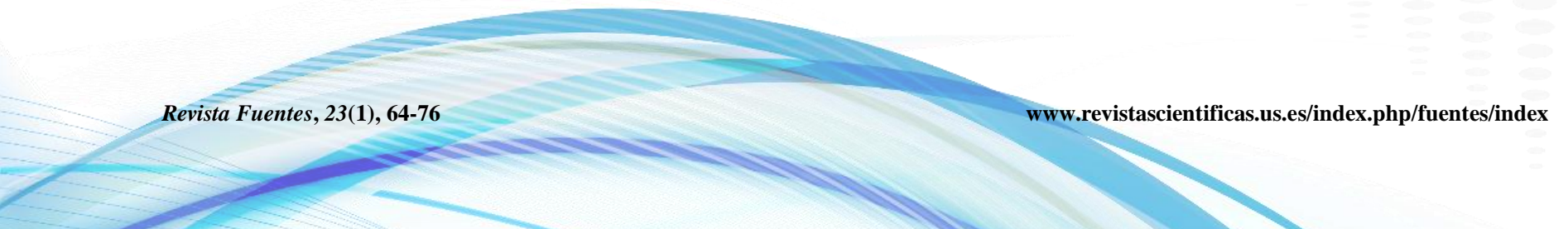

